III.

Aus der chirurg. Klinik (Geheimrath Kïster) und dem path. Institut (Geheimrath Marchand) zu Marburg.

\title{
Ueber Cystofibrosarkome der Mamma mit epidermoidaler Metaplasie.
}

$$
\text { Von }
$$

Dr. B. Grohé.

jetzt Assistenzarzt der chirorg. Klinik zu Jena.

(Nit 1 Abbilthung im 'Text und Tafel IIr.)

Die Geschwülste der Brustdrïse sind seit langen Zeiten so genauen, z. Th. systematischen Untersuchungen unterzogen worden'), dass wir über den klinischen Verlauf, die Prognose und die pathologische Anatomie eine genaue Kenntniss besitzen.

Am häufigsten sind die Carcinome. Nach Billroth's Statistik machen sie 82 Proc. aller Mammatumoren aus. Die übrigen 18 Proc. vertheilen sich auf die verschiedenen gutartigen Geschwuilste.

Schimmelbusch (l. c.) befand sich allerdings im Irrthum, als er Cruveilhier das Verdienst beimass, der erste gewesen zu sein, "welcher die Existenz gutartiger Tumoren der Mamma mit klarem Blick erkannte“. Zur Erläuterung erzählt er (S. 102), „dass Nichts bezeichnender für die Anschauungen der Zeitgenossen Cruveilhier's sei, als dass dieser mit dem Vortrag seiner Meinungen 1844 in der Académie de médecine in Paris einen wahren Sturm des Widerstandes hervorrief und in nicht endenwollenden Debatten überall ihm Opposition gemacht wurde".

Nein, wir brauchen nicht zu ausländischen Autoren unsere Zuflucht zu nehmen. Vielmehr hatte schon geraume Zeit vorher unser Johannes Müller die scharfe Unterscheidung der gut- und bösartigen Mammageschwülste ausgesprochen. ${ }^{2}$ )

1) Ich nenne neben Billroth: Die Krankheiten der Brustdrüse. Deutsche Chirurgie. Lieferung 4. 1880, nur die Arbeiten von Schimmelbusch, Arch. f. klin. Chirurgie. Bd. XLIV. 1892. Häck el, Ebend. Bd. XLVII. 1894. Sasse, Ebend. Bd. LIV. 1897.

2) Joh. Müller, Ueber den feineren Bau und die Formen der krankhaften Geschwülste. Berlin 1835. 
Bereits 1838 hat er die deutliche Abgrenzung des Cystosarcoma phyllodes von den Carcinomen gemacht, "welche so weit (in ihrer Gutartigkeit) vom Carcinom entfernt als jene blumenkohlartigen nicht eiternden Condylome des Penis und der weiblichen Geschlechtstheile, die so oft schon für Carcinome angesehen worden" (S. 53). Ja er widerruft sogar die Rubricirung zweier Mammatumoren, die er im Archiv für Anat. und Physiol. (III. Bd. 1836; Jahresbericht p. CCXII) als Carcinoma phyllodes beschrieben, indem er deren Gutartigkeit nach Kenntnissnahme der Krankengeschichten und genauer Untersuchung erkannte und sie dem Cystosarkom zuschrieb.

Die Formen der gutartigen Geschwïlste sind nun die mannigfaltigsten. Wir kennen Fibrome, Fibroadenome, Cystoadenome, Cystosarkome und die verschiedenen Zwischenglieder zwischen den genannten, welche die verwickeltsten Combinationen ergeben, ganz ahgesehen von den seltenen Fällen von Osteomen, Chondromen und ähnlichen Raritäten.

Bei der Gruppe der Cystosarkome sind nun auch relativ selten besondere Gewebsbildungen beobachtet worden, ich meine das Vorkommen epidermoidaler Structur im Tumor.

Der geringen Zahl der bisher bekannten Fälle kann ich im Folgenden zwei neue hinzufügen.

Der erste Fall wurde in der ehirurgischen Klinik zu Marburg. von Herrn Geheinrath Küster operirt.

Die Krankengeschichte ist folgende:

Anamnese: Patientin ist 27 Jahre alt, hereditär nicht belastet, früher war sie stets gesund. Vor 8 Jahren hat sie geboren; die Geburt ging leicht von statten; die Patientin stillte das Kind 8 Wochen lang; darauf nahm sie Stellung als Amme, in welcher sie 2 Jahre verblieb und 2 Kinder stillte. Während dieser ganzen Zeit war sie beschwerdefrei. Errst ein Jahr, nachdem sie die Stelle als Amme aufgegeben hatte, bemerkte sie in ihrer rechten Brustwarze einen kleinen Knoten. Irgend welche Schmerzen wurden durch den letzteren nicht hervorgerufen. Der Tumor nahm von ihrem 22. Lebensjahr (3 Jahre nach der Entbindung) stetig an Grösse zu und belästigte sie nunmehr etwas durch seinen Umfang. Lediglich aus diesem Grunde sucht sie die Klinik auf.

Status praesens: Patientin ist schlecht genährt, sieht älter aus, als es ihrem Alter entspricht. Innere Organe gesund.

Die linke Brustdrise ist wenig entwickelt, schlaff und welk. Die rechte Mamma weist etwa Kindskopfgrösse auf. Die Haut ist ohne besondere Veränderung; in derselben bemerkt man einzelne erweiterte, geschlängelte Venen. Die Form des Tumors ist rundlich, es heben sich an einzelnen Stellen flach gewölbte Prominenzen hervor. Die Geschwulst fühlt sich im Ganzen weich an; die erwähnten prominenten Stellen ergeben Fluctuation. Die Haut ist über dem Tumor verschieblich; dieser 
selbst ist mit dem Pectoralis nicht verwachsen. Weder in der Achselhöhle noch in der Supraclaviculargrube sind Drisenanschwellungen zu constatiren.

Die Operation bestand in der Wegnahme der ganzen Brustdrüse; der Verlauf der Heilung war in keiner Weise gestört.

Pathologisch-anatomischer Befund: Der Tumor hat die Grösse einer Männerfaust; er liegt im Fettgewebe eingebettet. Auf dem senkrechten Durchschnitt, der durch die Mamilla gelegt ist, sieht man, dass er von der normalen Mamilla und der bedeckenden Haut mit dem subcutanem Fettgewebe durch eine derbe Bindegewebskapsel scharf abgegrenzt ist. Von normaler Drïsensubstanz ist makroskopisch nichts mehr zu erkennen.

Des Weiteren bietet sich auf den Durschschnitten ein buntes Bild von kleineren und grösseren Cysten, in deren Lumen vielgestaltige, kurze, breitbasig aufsitzende oder langgesticlie, papilläre Wucherungen hineinragen. An einigen Stellen liegen diese so dicht gedrängt, dass sie sich gegenseitig comprimiren und den Cystenhohlraum ganz ausfutlen.

Eine grosse Anzahl von nicht mit diesen Excrescenzen ausgeffiliten Cysten zeigen eine merkwürdige Ausfïllung mit einer weissen, perlartig glänzenden, wie hornigen Masse, die an vielen Stellen eine concentrische Anordnung erkennen lässt. Sie liegen zum Theil in grösseren Partieen zusammen; dann scheinen sie hänfig die bindegewcbigen Septen zum Schwund gebracht zu haben und bilden so eine grössere confluirende Masse, deren ursprüngliche Znsammensetzung aber durch die verschiedenen concentrischen Structuren schon makroskopisch erkenmbar ist.

Danı finden sich diese Perlkugeln auch ganz vereinzelt in ihrer reinen Weisse scharf gegen die Umgebung abstechend, zuweilen in einer der erwähnten Papillen eingelagert, dann in den ditnneren oder dickeren Septen zwischen zwei Cystenrüumen. Jhre Grösse variirt dabui von Haselnussgrösse bis za den feinsten Pänktchen. In besonders leuchtend weisser Färbung treten sie an einzelnen Stellen auf, wo ansgedehnte Blutungen in und zwischen die Tumormassen stattrefunden haben.

Die papillären Wucherungen, ans welchen sich im Uebrigen der Tumor zusammensetzt, bestehen zum Theil aus einem derben Bindegewebe. Ein grosser Theil ist aber ausserordentlich weich, von etwas glasigem Aussehen und macht völlig einen myxomatösen Eindruck. Diese Stellen sind es wohl auch gewesen, welche vor der Operation einen pseudofluctuirenden Cbarakter bei der Palpation aufwiesen.

Die peinlichste Schilderung des Tumors diurfte bei seinen variablen Bildern kaum eine so lebhafte Vorstellung ermöglichen, wie es durch seine zeichnerische Wiedergabe geschehen kann. Ich füge diese um so lieber bei, da die bisher existirenden Abbildungen kaum uns ein annähernd richtiges, uberzeugendes und anschauliches Bild von den in Frage stehenden Tumoren geben diurften. Der vorliegende Tumor dürte aber wohl als ein geradezu klassischer Typus der Reproduction werth erscheinen.

Fig. 1 auf Taf. III ist ein Schnitt senkrecht durch die Höhe der Mamilla gelegt. Zunächst ist die scharfe Abgrenzung des Tumors nach oben deutlich erkennbar. Nach unten ist er nicht so scharf abgegrenzt; seine Hülle bestand hier nur aus dunnen Bindegewebspartieen, so dass er sich mit Leichtigkeit vom Musc. pect. major abpräpariren liess. Wir erkennen die 
grösseren und kleineren vielgestaltigen Hohlräume und die hier besonders vereinzelt auftretenden, scharf abgegrenzten Perlkugeln.

Ein viel reichlicheres lild der mit verhornten Massen erfüllten Cystenräume liegt in Figur 2 vor. Der Schnitt ist ca. $3 \mathrm{~cm}$ weit von der Mamilla ebenfalls in verticaler Ebene zur Unterlage gelegt und bringt sowohl kleinere Cystendurchschnitte zur Anschauung wie auch die grösseren theilweise confluirenden Massen $(f)$ mit ihrer Vielgestaltigkeit.

Die mikroskopische Untersuchung, welche sich auf alle Gebiete des Tumors erstreckte und die mit verschiedenen Färbemethoden ausgefuthrt wurde, ergab folgende Bilder:

Die Grundsubstanz in den Präparaten besteht im Wesentlichen ans einem ziemlich kernreichen Bindegewebe, an anderen Stellen aus myxomatösen Partieen, welche stellenweise fast ganz kernlos werden und einem weitmaschigen, z. 'Th. mit zahlreichen Gefässen durchzogenen Gewebe Platz machen.

Dem makroskopischen Aussehen entspricht die Form des Schnittes dort, wo eine papilläre Wucherung getroffen ist, indem an den Grundstock knospenartige oder bucklige Fortsetzungen sich anschliessen, während lie und da die Querschnitte von diesen, dann natïrlich olne Zusammenhang mit dem übrigen Präparat, sich finden.

Die epitheliale Bekleidung zeigt nun die verschiedensten Charaktere.

An einzelnen Stellen, besonders dort, wo der myxomatöse Ban vorwiegt, findet sich ein cubisches, ein-, zuweilen auch zweischichtiges Epithel, welches hie und da einem hohen Cylinderepithel Platz macht. Ist dieses meist mehrschichtig, so sieht man an einzelnen Stellen, wo es sich als schmale Schläuche oft tief in das Gewebe hineinsenkt, eine zweireihige Anordnung, zu oberst eine Cylinderform, darunter eine ebenso regelmässig angeordnete mehr cubische. Man vermisst unter diesen aber eine Membrana propria. Das Fehlen dieser kann man gut controliren an Stellen, wo in dem Grundgewebe sich vereinzelt Quer- oder auch Längsschnitte derartiger Canäle finden, deren Epithelauskleidung oft ohne besondere hervorstechende Grenze in das umgebende zellreiche Gewebe übergeht. ${ }^{1}$ )

Bei der weiteren Verfolgung des Epithelsaums verbreitert sich stellenweise das mehrschichtige Cylinderepithel allmählich immer mehr und mehr und ohne scharfe Grenze setzt dann eine Bedeckung mit geschichtetem Pflasterepithel ein, völlig dem Typus der Epithellage der äusseren Haut entsprechend. Die Basalschicht, scharf von der Unterlage abgesetzt, verläuft in gerader Linie, ohne Einsenkungen wie bei den Papillarkörpern der normalen Haut. Sie besteht aus langen, palissadenartig angeordneten Zellen, daran reihen sich die cubischen, die ovalären Formen, in welchen man deutlich Riffzellen sieht und schliesslich bilden den Beschluss die der Verhornung zugehenden mit Eleidinkörnern versehenen platten Zellen, auf welche total verhornte, kernlose Massen aufgelagert sind.

Diese epidermoidale Schicht setzt sich nun theilweise in langer Linie fort, bis sie ganz allmählich dem Eingangs geschilderten cylindrischen Epithel wieder Platz macht. ${ }^{2}$ ) Zuweilen sieht man sie auch scharf unter-

1) Ich controlirte diese Befunde num so gemaner, da Kürsteiner hierauf speciell hinweist.

2) Diese Bilder entsprechen völlig denen, welche G. B. Sc h midt in der Fig. 4 der Tafel II sciner Arbeit darstellt. 
brochen durch eine kleine drüsenschlanchartige Einstïlpung, welche eine einschichtige, schöne Cylinderepithelauskleidung besitzt.

Eine grosse Reihe von Cysten weist aber nicht eine solche Mannigfaltigkeit auf, sondern besitzt lediglich eine epidermoidale Auskleidung, deren Mächtigkeit und Anordnung den verschiedensten Schwankungen unterworfen ist. An einzelnen Partieen findet sich eine ganz ansehnliche Dicke der Epidermis, an anderen Stellen nimmt sie sehr an Mäch-

Fig. 3. Ṡchnitt durch eine papilläre Excrescenz mit Epitholperteneinlagerung ivergl. s. 71\%. - Lapenvergö̈serung $3_{j}$.

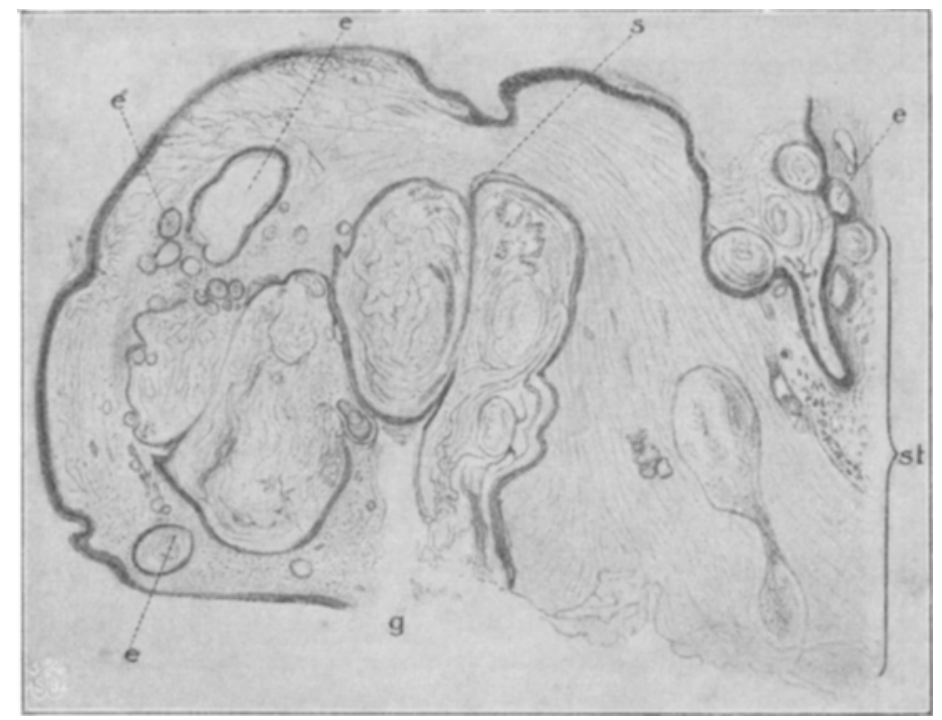

$s t=$ Stiel der Wucherung. $-e=$ Fpitholperlen gefüllt mit verhornten Massen. $s=$ ein Septum zwischen zwei Cysten zim Theil atrophirt. - $g=$ gemeinsamer Ausführmesgang der Cysten.

tigkeit ab. Ihre wandbekleidenden Zellen scheinen oft wie abgeplattet durch den Druck, welchen die verhornenden und verhornten Massen im Innern der Cyste ausuben. Liegen mehrere derartige Cysten zusammen, so sind ihre Septa oft ganz dünn geworden, an einzelnen Stellen sind sie völlig geschwunden und nur noch Reste wie coulissenartige Seitenwände bezeichnen die frühere Trennungswand. Dadurch kommen dann die confluirenden Cysten zu Stande, wie sie in Fig. 3 (siehe oben) ersichtlich sind. Diese Abbildung giebt uns überhaupt in ihrer Lupenvergrösserung eine Ansehaung, wie der Aufbau in den uns interessirenden Tumorabschnitten sich gestaltet. Der Schnitt selbst stellt eine papilläre Excrescenz in einem grösseren Hohlraum dar. Er ist, abgesehen von dem glatten Stiel (st), von Plattenepithelien bedeckt. In seinem Inpern finden sich kleinere und kleinste Cysten, solann grössere, welche z. Th. in einander übergehen und einen gemeinsamen Ausführungsgang $(g)$ besitzen. Das Stroma in 
diesem Schnitt ist ein ziemlich derbes, kernames Bindegewebe, welches stellenweise von dilatirten Gefässen durchsetzt ist.

Bei der Durchmusterung weiterer Präparate finden sich schliesslich auch vereinzelt im Gewebe liegende Cysten, welche nur noch theilweise ihre epidermoidale Wandbekleidung aufweisen. Stellenweise siebt man die Epithelien zerfallen, freie Kerne oder Kernrudimente bilden mit total nekrotischen Gewebspartieen eine, die Färbung nur noch undentlich annehmende Masse. Solche Nekrosen der Cysten finden sich sogar fernerhin noch in ihrer ganzen Totalität. Dort schliessen sich an ein ziemlich kernarmes Bindegewebe, in dem einzelne Drüsenlumina und solide epitheliale Zellstränge liegen, cystische Partien an, deren Ränder ein abscessartiges Aussehen besitzen. Von einer epithelialen Auskleidung ist nichts mehr zu erkennen, vielmehr wird der Rand der ehemaligen Cyste ringsum umgeben von Bindegewebe, welches minimale Drüsenreste in sich birgt. Auf das Bindegewebe folgt nach innen zu ein gefässreiches Gewebe. Längs der Capillaren liegen Zellen mit grossen, ovalen Kernen, dazwischen sind einige Mitosen sichtbar. Beim weiteren Vorgehen tauchen (bei Hämatoxylin blau tingirte) bomogene kleinere und grössere Schollen anf. An diese sind Zellen angelagert, welche nach Grösse und Form den vorhin erwähnten entsprechen. Dazwischen liegen melırkernige grössere Zellen, bei denen der Theilungsprocess sich vorerst nur auf die Kerne beschränkt hat und der Zellleib sich nur in toto vergrössert, aber nicht selbst in Theilung übergegangen ist. Riesenzellen mit zahlreichen Kernen findet man ausserdem auch in einiger Entfernung von den Schollen. Wenn wir nun die homogenen Schollen und die Riesenzellen zusammengelagert sehen, so ist wohl der Gedanke nicht abzuweisen, dass beide in einem gewissen Zusammenhang stehen und diese als Fremdkörperriesenzellen anzusehen sind.

Haben wir somit die uns vornehmlich interessirenden Stellen des Tumors kennen gelernt, so mïssen wir schliesslich noch erwähnen, dass wir einzelne Stellen im Tumor haben, wo das gewöhnliche Bild des Cystosarkoms resp. Fibroadenoms zu Tage tritt. Es sind Partieen, welche den mehr als homogen derb geschilderten Gebieten des Tumors entstammen. Wir sehen die kürzeren oder längeren Drüsenschläuche mit einsehichtigem Cylinderepithel bedeckt. Stellenweise sind sie dilatirt; zwischen ihnen findet sich ein ausserordentlich zellreiches Sarkomgewebe vorwiegend von Spindelzellenstructur, untermischt mit einer Zwischensubstanz von fibrillärer Anordnung, in welcher sich hie und da sehr reichliche Mitosen finden.

An der einen schon makroskopisch als Blutung imponirenden Stelle sind diese Drüsenschläuche getrennt durch reichliche Blutextravasate, welche in das $Z$ wischengewebe vordringen und es a us inande $\mathrm{rged} r$ äng t haben. Normales Drüsengewebe war an keiner Stelle der amputirten Mamma nachweisbar.

Resumiren wir kurz den mikroskopischen Befund, so haben wir es in der Hauptsache zu thun mit einem cystosarkomatösen Tumor, dessen Cysten stellenweise die übliche Auskleidung mit cylindrischen Zellen besitzt. An anderen Stellen tritt ohne scharfe Grenze eine allmähliche Umwandlung dieser Zellformen in typische Plattenepithelien 
ein. Dieselbe kann aber auch in scharf abgesetzter, unvermittelter Form auftreten. Einzelne Cysten erweisen sich schon völlig von Plattenepithelien ausgekleidet. Diese sind meist an ihren oberen Schichten stark verhornt und als Product dieses Processes sieht man die Cysten völlig vollgepfropft mit verhornten Massen, welche makroskopisch perlartige Bilder veranlassen.

Als ich mit der Bearbeitung dieses seltenen Tumors beschäftigt war, wurde zufälligerweise ein zweiter ganz älnlicher Tumor von Herrn Prof. von Büngner aus dem Hanauer Landkrankenlıause dem pathologischen Institut zu Marburg iibersandt. Herr Geheimrath Marchand, der mich bei dieser Arbeit rege durch Rath und That gütigst unterstützte, lıatte die Liebenswürdigkeit, mir diesen Tumor zur Mitbearbeitung zu überweisen.

Die von Herrn Prof. von Büngner freundlichst übermittelte Krankengeschichte lautet:

Frau P., 48 Jalre alt, aus Hanau, ist vor Beginn ihres jetzigen Leidens stets gesund gewesen. Vor etwa 4 Monaten bemerkte sie, dass ihre linke Mamma an Umfang zunahm. Eine Ursache dafür weiss Patientin nicht anzugeben. Ein Trauma hat sie nie erlitten, nie eine Entzitndung der Mamma durchgemacht. Seit einigen Wochen sind in der erkrankten Brustdrüse, welche sich immer melhr vergrösserte, ziehende und stechende Schmerzen aufgetreten.

Status: Die linke Mamma ist in einen starren, kindskopfgrossen Tumor umgewandelt, der an der Oberfläche einige Höcker und an verschiedenen Stellen Fluctuation zeigt. Haut und Mamilla lassen sich uber dem Tumor nicht verschieben, während der Tumor in ganzen auf der Unterlage verschieblich ist. Die regionären Iymphdrilsen sind nicht geschwollen.

Die exacte Diagnose der Art des Tumors muss der histologischen Untersuchung vorbehalten bleiben. Klinische Wahrscheinlichkeitsdiagnose: Cystosarcoma mammae.

2. Juni 1898: Amputation der Mamma mit Entfernung der Fascie des Muse. pect. major und Eröffnung der Axilla. In der typisch ausgeräumten Achselhöhle keiue erkrankten Lymphdrüsen. Nahtvereinigung der Wunde. Drainage.

Heilverlauf ohne Störung. Prima intentio. Entlassen 13. Juni 1898.

Die makroskopische Betrachtung des Tumors ergiebt, dass unter der normalen Haut und der gut entwickelten Mamilla sich ein eirca 2 Faust grosser Tumor findet. Auch er ist von der Umgebung scharf abgegrenzt; an vielen Punkten, so von der bedeckenden Haut durch ein gut entwickeltes Fettpolster getrennt.

Der Tumor selbst setzt sich zusammen aus einer grossen Anzahl von Cysten und Knollen. Letztere, welche in ihrer Compactheit hier weit mehr überwiegen wie bei dem ersten Tumor, zeigen eine sehr verschiedene Beschaffenheit. Einige sind sehr solide und atus derben, ziemlich homo- 
genen, weisslichen Gewebsmassen zusammengesetzt. Andere bestehen aus charakteristischem Schleimgewebe, wieder andere zeigen eine derbe, faserige Structur.

Auch hier finden sich nun in das Gewebe eingesprengt, und zwar wenn wir uns den Tumor durch zwei auf die Körperoberfläche senkrecht gelegte Ebenen in drei gleichmässige Theile zerlegt denken, lediglich in dem äusseren Drittel des Tumors bis bohnengrosse, runde, scharf abgegrenzte Cysten, welche ebenfalls mit einem perlartigen Material gefültl sind und eine concentrische Anordnung dieser Massen zeigen. Derartige epidermoidale Massen sind auch vielfach dem flitssigen Inhalt beigemischt, der aus einzelnen Cysten sich entleert und massenhaft glitzernde Cholestearinkrystalle enthält. Einige Cysten enthalten auch hämorrhagischen Inhalt, während ein grosser Knoten im Centrum völlig bämorrhagisch erweicht ist und eine unregelmässige Höhle darbietet. Die nicht erweichten angrenzenden Theile sind stark mit Blut durchsetzt und zeigen eine theils bräunliche, theils dunkelrothe Farbe.

Sonach unterseheidet sich schon makroskopisch dieser Tumor in einem grossen Theil seiner Structur von dem erstgeschilderten, indem er im wesentlichen die Bilder des Cystosarcoma phyllodes (Muller) s. Myxoma papilliforme intracanaliculare (V i $\mathrm{chow}$ ) bietet. Diesem makroskopischen Verhalten entspricht nun auch in vielen Punkten der mikroskopische Befund.

Zunächst sei bemerkt, dass sich unter der Haut, in dem zwischen ihr und den eigentlichen Tumormassen liegenden Fettgewebe, aber auch noch an einzelnen mehr in der Tiefe der Geschwulst und zwar an den peripherwärts gelegenen Stellen normales mamilläres Drüsengewebe findet.

Die geschilderten derben zum Teil blättrig angeordneten Partien zeigen die bekannten Bilder des Cystosarcoma phyllodes proliferum. Lange schmale Canäle, die in ausgedehntester Weise Ausläufer hierhin und dorthin senden, verbinden sich schliesslich zu grösseren Gängen, stellenweise sich zu spaltförmig-cystischen Räumen erweiternd. Die Wände der Canäle werden gebildet aus einem meist mehrschichtigen cubischen, an einzelnen Stellen in seinen obersten Schichten cylinderförmigen Epithel. Die durch die Spalten und Cysten papillenartig gestaltete Grundsubstanz des Tumors besteht aus einem Gewebe, welches im wesentlichen den Typus des Spindelzellensarkoms repräsentirt. An anderen Stellen geht es jedoch über in ein zellarmes Myxomgewebe, welches bei Hämatoxylinfürbung durch seine blassviolette Fïrbung in die Augen sticht. Versehiedentlich ist das Gewebe durch zum Theil sehr ausgedehnte Blutergüsse auseinandergedrängt, in deren Umgebung dann stellenweise ein Zugrundegehen des Gewebes stattgefunden hat, so dass von einer normalen Kernfärbung überhaupt nicht mehr die Rede sein kann.

Neben diesen Bildern besitzen wir, wie erwähnt, noch derbere Knoten von festerer Consistenz in der Mamma, welche mikroskopisch lediglich den Bau eines reinen Spindelzellensarkoms zeigen. Dieselben setzen sich vollständig zusammen aus grossen Spindelzellen, die an den Rändern der Knoten ziemlich scharf von derbem Bindegewebe umgeben sind, aber auch hier und da langsam streifenförmig in dasselbe hineingewuchert sind und dadurch in Verbindung mit danebenliegenden gleichartigen Gewebsmassen stehen. 
Während die bisher geschilderten Bilder sich in ungefähr $2 / 3$ des Tumors finden, ändert sich der Befund, wenn wir uns jener Partie des Tumors zuwenden, welche schon makroskopisch durch das Auftreten von perlartig glänzenden Cysten unsere besonderc Aufmerksamkeit auf sich zog.

Die eben beschriebenen cystischen Ränme besitzen nämlich nicht mehr ausnahmslos ihre cubisch-cylindrische Epithelauskleidung; es tritt vielmehr an ihre Stelle ein mehrschichtiges Plattenepithel. Die Zelllagen besitzen eine verschiedene Mächtigkeit. Die Basalschicht weist meist eine palissadenartige Anordnung auf, darauf bauen sich - ähnlich wie bei der äusseren Haut - mehrere Lagen rundlich-ovaläre Zelien, die mit feinen zahlreichen Stacheln besetzt sind. Diese Zelllagen platten sich mehr und mehr ab, die Kerne werden spärlicher, in einzelnen Zellen finden sich feinste Körnchen, und schliesslich haben wir weiter central vollständig verhornte, zwiebelschalenartig gelagerte Massen, die in den grösseren Hohlräumen zu einem ungeordneten Brei zusammengeballt sind.

Eine Andeutung von Papillarkörpern unter dem Rete Malpighi haben wir auch hier nirgends. Die Mächtigkeit der epidermoidalen Schicht variirt ausserordentlich. In einzelnen Cysten kann man eine Aufeinanderlagerung bis zu 10 Zellreihen zählen, wobei die verschiedensten Uebergangsformen zu studiren sind, während in anderen Cysten die Epithelschicht eine ganz niedrige ist, zuweilen eigentlich nur aus zwei Lagen besteht, so dass die verhornten Massen sich direct den beiden Zelllagen anschliessen. Auffallend gerade an diesen Stellen ist es, dass wir nirgends den Keratinkörnchen einer La n ge rhans'schen Körnerschicht begegnen: ein Beweis, dass eine derartige Körnung durchaus keine Vorbedingung für die Verhornung ist. Endlich finden wir Cysten, wo eine geregelte Epithelanordnnng resp. -auskleidung völlig fehlt; die homogenen Platten und Schollen liegen direct dem bindegewebigen Stroma auf, höchstens dass sich noch hier und da einige wandständige Epithelien finden. Die Form dieser Räume ist auch nicht mehr regelmässig wie die der anderen, sondern sie ist buchtig oder vieleckig geworden.

Diese Variationen diurfen wir wohl auf Compressionsfactoren zurückführon, welche bei der Bildung des Tumors sich abgespielt haben. So kann es sich um Compression von innen heraus handeln, indem die immer reichlicheren verhornten Massen ad maximum den Hohlraum ausfüllen und dadurch die Epithelschichten in ihrer Entwicklung gehemmt resp. zurïckgedrängt werden.

Aber gleich der epithelialen Auskleidung zeigt auch der ubrige Theil der Cystenwandung wiederholt grosse Verschiedenheiten. Während nämlich das eine Mal eime starke, oft hochgradige Wucherung des bindegewebigen Antheils constatirt werden kann, erscheinen bei anderen Cysten die Wandungen geradezu atrophisch. Diese Erscheinung kann nun ebenfalls auf die weiter schreitende Ausdehnung der Cysten durch Verhornungsmassen zurückgeführt werden. Wir hätten es dann mit einer Druckatrophie der Cystenwandung zu thun, und dies erscheint mir am wahrscheinlichsten. Man ist aber anch berechtigt, an eine bedeutende Compression von Seiten der mächtig wuchernden bindegewebigen Nachbarschaft zu denken. Wir haben es hier mit derselben Frage zu thun, ob das Epithel 
oder das Bindegewebe das primäre Agens ist, wie bei der Anlage der papillären Excrescenzen in cystischen Räumen.

Ohne hier auf diese Frage näher eingehen zu wollen, sei nur erwähnt, dass wir in einzelnen Cysten unseres Tumors Bilder finden, wo die epitheliale Auskleidung - und zwar handelt es sich hier um ein cubisches Epithel -- kleine knöspchenartige Hervorragungen zeigt, welche aus einem grösseren Convolut von Epithelien bestehen. Sie weisen darauf hin, dass wir es mit den ersten Anlagen zur Neubildung von papillären Wucherungen zu thun haben. Als Hauptsache der Weiterbildung ist aber nach unserer Anschaunng die Wucherung des Bindegewebes anzusehen, wie es ja auch bei dem einfachen Fibro-Adenom immer die Drüsensclıäuche umgiebt.

Der Vollständigkeit halber soll nicht unerwähnt bleiben, dass in einigen Cysten, speciell denen, welche eine Keratinbildung in den verhornenden Schichten zeigen, in den obersten der letzteren in einzelnen Zellen neben dem Kern rundliche, blass tingirte, bläschenartige Gebilde - sie treten besonders bei van Gieson's Färbung schön hervor - liegen, welche ich nicht anstehe als Zerfallsproducte von Zell- resp. Kernsubstanz anzusehen. Forscher, welche das Gebiet der Zelleinschlusse bei Tumoren besonders berlicksichtigen, wïrden vielleicht vermuthen, dass es sich um parasitäre Gebilde handle.

Resumiren wir die Hauptbefunde auch bei diesem 'Tumor, so haben wir es im wesentlichen mit einem ausgresprochenen Cystosarcoma phyllodes zu thun; nicht ganz so mächtig ist das Vorkommen eines reinen Spindelzellensarkoms zu verzeichnen. In einem Drittel des Tumors haben wir in diesem Gewebe schon makroskopisch sichthare, theils ineinander übergehende. Cysten mit perlartig olïnzendem Inhalt.

Mikroskopisch sehen wir in diesen Partien noch an einzelnen Cysten Auskleidung mit cubischem Epithel, während die Mehrzahl derselben mit mehrsehichtigem Plattenepithel ausgekleidet ist, das in den oberen Schichten in Verhornung ïbergeht. Dieser Process hat sich in vielen Fällen so mächtig abgespielt, dass die IIollläume völlig mit verhornten Partien erfüllt sind, so dass die makrokopisehe perlartige Ausfïllung resultirt.

Zwar nicht wie in Tumor I finden sich hier so ausgesprochene, schöne Bilder der verschiedenen Epithelarten nebeneinanderstehend; wir haben hier vielmehr wohl weit vorgeschrittene Stadien mit älteren Verhornungsprocessen vor uns, und nur an spärlichen Stellen ist es noch möglich za studiren, wie die Metaplasie der Epithelien sich abgespielt hat.

Soweit unsere Fälle! Betrachten wir nunmehr die hisher bekannten Fälle derartiger Geschwülste, so sind die letzten ausführlichen Beschreibungen niedergelegt in zwei Arbeiten, welche beide im Jahre 1894 erschienen sind, und zwar von II ä ckel und Kürsteiner.' Besonders 
der erstere Autor hat eine fast erschöpfende Aufführung der publicirten Fälle gegeben. Ich konnte noch einige ergänzend hinzufügen und führe die mir nunmehr bekannten auf, indem ich theilweise ihre genaueren Befunde berichte, da ich glaube, dass man bei ihrer Gegenüberstellung manche Punkte besser beleuchten und erörtern kann, unsomehr, da die Ansichten und Deutungen in manchen Punkten nicht dieselben sind.

Abgesehen von Porta ${ }^{1}$, welcher im tieferen Zellgewebe der Brustdrüse 3 ,Tumores sebacei" aufzählt, ohne dass wir über dieselben genauere Details erfahren, beschreibt den ersten einschlägigen Fall A. Cooper ${ }^{2}$ ) als eine Hydatidensackgeschwulst kugeliger Natur. Es handelt sich um einen 35 engl. Zoll im Umfang messenden Tumor, welcher nach einem Stoss bei einer 44 Jahre alten Frau entstanden, besonders in den letzten Jahren rasch gewachsen und im 14. Jahre seines Bestehens exstirpirt wurde.

Der Tumor bestand aus theils derben Gewebemassen, theils "serösen Säcken", in welche büschelförmige, polypöse Wucherungen hingen. Ihre Grösse war höchstens die eines Gerstenkorns. „Wenn sie geöffuet wurden, zejgte sich, dass sie aus zahlreichen Schichten zusammengesetzt waren, wie die Linse im Auge oder wie die Hänte einer Zwiebel, welche leicht von einander abgeschält werden können. Als sie aus der Brust herausgeuommen wurden, hatten sie das Aussehen und auch die geschichtete Anordnung der Perlen".

Eine Schilderung des mikroskopischen Befundes fehlt, doch die Abbildungen der Tafel III und IV, Nr. 1-10 geben den deutlichen Beweis, dass wir es mit einem Tumor unserer Art za thun haben.

Virch ow ${ }^{3}$ ) erwähnt in seiner Arbeit „Ueber Perlgeschwülste“, dass er in Brustdriisengeschwülsten, namentlich in Cystosarkomen, wiederholt Cholesteatom-Perlen gesehen babe, doch immer in kleiner Anzahl.

Ihm zur Folge hat auch $\mathrm{Bruch}^{4}$ ) einen Fall von Combination eines Brustdrüsenkrebses mit kleinen Cholesteatomperlen beschrieben. Er soll ganz ähnlich dem Cystosarkom von $\mathrm{M}$ ïll $\mathrm{er}^{5}$ ) sein, welches ebenfalls Cysten mit epidermisartigen Epithel aufwies.

Die erste genaue Untersuehung eines derartigen Tumors lieferte nun Model $\left.{ }^{6}\right)$ :

Es handelt sich um eine 37 jährige Frau, die 12 Jahre verheirathet war und ein Kind nur rechts gestillt hatte. Vor 3 Jahren hatte sie das Entstehen einer kleinen beweglichen Geschwulst bemerkt. Als dann eine

1) Porta, Memoria letta all' i. r. istituto lombardo di scienze, lettere ed arti. Ref. Schmidt's Jahrb. 1857. Bd. XCVI.

2) A. Cooper, Darstellung der Krankheiten der Brust. Dentsche Uebersetzung. Weimar 1836 . S. 16.

3) Virchow's Archiv. Bd. VIII. 1855. S. 399.

4) Bruch, Diagnose der bösartigen Geschwülste. S. 49.

5) J. Müller, Ueber den feineren Bau der krankhaften Geschwülste. 1938. S. 58 .

6) Aug. Model, Ein Fall ron glandulärem Cystosarkom mit autogenem Atherom. Ing.-Diss. Erlangen 1858. 
Kuh mit dem Horn gerade gegen die Geschwulst gestossen hatte, war in einem halben Jahre ein rasches Wachsthum mit geringen Schmerzen eingetreten.

B ef und: Doppelfaustgrosser, nirgends adhärenter Tumor mit vielen Höckern, die rundlich und glatt anzufühlen sind, die kleineren sind sehr derb und resistent, die grösseren, die den Umfang einer Wallnuss erreichen, sind weich und elastisch, einer mit dunkler Fluctuation.

Der exstirpirte, cystenartige Tumor besteht aus glandulär entartetem, zum Theil atrophirtem Brustdriisengewebe. Die Mehrzahl der dabei gebildeten cystenartigen Gebilde ist mit perlartigen Körperchen erfüllt von den minimalsten Entwicklnngsstufen bis zu grösseren, im Innern erweichten Höhlen. Diese atheromartigen Bälge sind unregelmässig durch die entarteten Drüsen verbreitet und im Stroma zerstreat.

Die kleineren der atheromartigen Gebilde stellen darch ihre eigenen Contenta oder die hineinwuchernde Drüsengewebsneubildung ausgedehnte Säcke dar; die grösseren verwickelteren Räume sind durch Verschmelzung entstanden.

Mikroskopisch zeigen diese Cysten alle eine epidermoidale Auskleidung.

Model nimmt an, dass nur manche Perlen die Fähigkeit zu besitzen seheinen, durch fortgesetzte Production von Epidermiszellen sich zu cystenartigen Gebilden zu entwickeln, während andere in der Entwicklung stehen geblieben und nur grosse Epidermiskugeln sind, welche in dem Stroma eingebettet liegen; sie stellen eine atrophische Form dar, während man bei den anderen eine producirende, fortwährend junge Zellen nachbildende Wand erkennt.

Die A nfäng e der perlähnlichen Epidermisbildungen sollen in Wucherungen liegen, die von den zelligen Elementen der Bindesubstanz ausgelien; eine Auffassung, welche sich durch die damaligen pathologischanatomischen Auffassungen erklärt.

Der Verfasser vergleicht sie mit den Cholesteatomen (s. Margaromen s. Margaroiden) und nennt sie, da sie sich schwer von den gewöhnlichen, durch Ektasie entstandenen Atheromen uuterscheiden, ausser durch ibren selbständigen Ursprung, der sich eben an Orten findet, wo normaler Weise keine Epidermialgebilde auftreten oder nicht hinwachsen können, a u togene Epidermisperlen resp. autogene Atherome.

Nur eine kurze Beschreibung liefern fernerhin Bauchet und Gailletl) von einer grossen Zahl kleiner Cysten vom Volumen eines Roggenkorns oder einer Erbse von weisser Farbe und fester Consistenz, ähnlich einer durch Congelation gehärteten Krystallinse, und ebenfalls cursorisch werden von $\mathrm{Neumann}{ }^{2}$ ) Cysten mit geschichtetem epidermisartigem Epithel erwähnt.

Die Existenz derartiger Tumoren im Allgemeinen constatirt K lob 3 ), dem zufolge (p. 499) „die Cystosarkome der Mamma in manchen Fällen accidentelle Gewebsentwicklungen zeigen, von welchen nament-

1) Gaz. des hôpitaux. 1861.

2) Neumann, Zur Casuistik der Myxome und Cylindmone. Areh. f. Heilkunde. Bd. IX. S. 480.

3) Jul. M. Klob, Path. Anatomie der weiblichen Sexualorgane. 1864. 
lich Cholesteatommassen und Enchondrome zu erwähnen sind. Die ersteren finden sich als erbsen- und bohnengrosse, weisse, glänzende, eingebalgte Körner oft in bedeutender Anzahl". Im Speciellen (p. 507) fand er in $z w$ e $\mathrm{F}$ ällen von exquisitem Medullarcarcinom der Mamma ganz kleine bis erbsengrosse, weisse, blättrige, runde Cholesteatome zerstreut in die Krebsmassen eingetragen.

In seiner Monograplie der Mamma erwähnt Billroth ${ }^{1}$ ) bei den proliferen Cystosarkomen (p. 61) nur kurz, dass bei diesen Tumoren , selten das Vorkommen einfacher, runder Cysten, noch seltener das von weissen Kngeln (Epithelperlen) in den Cysten ist".

Ich weiss nicht, ob er lierbei an den im Pitta-Billroth'schen Handbuch der Chirurgie III, 1865 als Cancroid beschriebenen Tumor dachte, welcher sich bei einer 40 jährigen Frau binnen einem halben Jahre unter den gewöhnlichen Erscheinungen des Krebses bis zur Faustgrösse entwickelte. Auf seinem Durchschnitt zeigte sich eine grosse Menge von hirsekorngrossen, mit weisslichem Brei gefullten Höhlungen, die er als erweiterte Acini ansah. Der Inhalt bestand aus dichtgedrängten, polyedrischen und geschwänzten Zellen. Derselbe findet sonst in besagter Monographie keine Erwähnung.

Ob wir za der Gruppe unserer Tumoren den von Bill roth (p. 90) geschilderten Fall hinzurechnen können, erscheint mir zweifelhaft. Häckel führt ihn in seiner später zu erwähnenden Arbeit an, doch spricht er dort von Atheromen der Mamma. Billroth selbst wagt nicht zu entscheiden, ob die enteneigrosse Riesenperlgeschwulst, welche sich als exquisites Specimen eines blättrigen Cholesteatoms erwies, aus einem Drusenläppchen der Mamma oder aus einer etwas tiefliegenden Talgdrüse der Haut hervorgegangen war, da die äusserlich wohlgeformte Brust zwar von Drtsensubstanz keine Spur mehr zeigte, das ganze Organ vielmehr aus Fettgewebe bestand und besagter Tumor dicht unter der Haut, ohne jedoch mit dieser verwachsen zu sein, sass.

Dass derartige Tumoren selbst in der Mamma des Mannes vorkommen, beweist das Epithéliome pavementeux corné lobulé du sein, welches von $\mathrm{Bruch}{ }^{2}$ ) einem 44 jährigen Manne nach 2 jährigem, langsamem Wachsthum aus der rechten Mamma exstirpirt wurde.

Die orangegrosse Geschwulst war ausserordentlich hart. Sie setzte sich makroskopisch zusammen aus einer Anzahl Knoten, die zum Theil von kleinen Cysten durchsetzt waren. In diesen oder in gewundenen Strichen finden sich Einlagerungen von knorpel- oder hornartigem Aussehen. Mikroskopisch beobachtete man eine theilweise Auskleidung mit Cylinderzellen; hauptsächlich aber waren es Plattenepithelien, welche oft in Epidermiskugeln zusammengeballt und völlig in verhornten Zustand ibergegangen waren.

Ich komme zu den drei letzten und auch ausführlichsten Special-

1) Billroth, Die Krankheiten der Brustdrüse. Dentsche Chirurgie. 41. Iieferung. 1950 .

2) Brreh, Bulletins et Mémoires de la Société de Chirurgic de P'aris. 1885. tome XI. p. 887. Vergl. D. Schuch ardt, Langenl. Ardh. Bd. XI.I. 1891. \$. 79) 
arbeiten auf unserem Gebiet, von denen zunächst die von G. B. Sch midt 1) zu nennen ist.

Er untersuchte ein Cystosarcoma mammae, in welchem sich mehr oder minder kugelige Cysten vorfanden, deren Wandung ein vollständiges Gepräge der äusseren Haut mit verhornten Producten darbot.

Der Tumor fand sich bei einer 72 jührigen Virgo, welche vor 3 Jahren von einer Freundin stürmisch empfangen und gegen die Brust gedrückt war. Sie behielt eine kaum merkliche Hmpfindung in der rechten Mamma zurück, deren sie sich noch erinnerte, als sie wenige Wochen danach eine kleine, harte, empfindliche Stelle in der Brustdriise bemerkte. Fast $21 / 2$ Jahre blieb die Geschwulst unverändert, dann wuchs sie ohne bekannte Ursache.

Die Geschwulst war aber den grössten Theil der nur kleinen Mamma verbreitet, flach, handtellergross, an der Oberfläche höckerig und von ungleicher Härte. Die am meisten hervorragenden Stellen fühlten sich elastisch, einzelne fluctuirend an. Die Haut war uber der Geschwulst und diese auf der Unterlage verschiebbar. Es bestanden keine Drïsenschwellungen.

Makroskopisch waren es kleinste bis kirschkerngrosse Hohlräume, welche mit einer weissgrauen, perlglänzenden Masse ausgefüllt waren, die concentrische Schichtung erkennen liess.

Mikroskopisch zeigte die Cystenwand zunächst der Peripherie ein einschichtiges, an anderen Stellen mehrschichtiges Cylinderepithel; dann folgte eine Lage polyedrischer, grosser Plattenepithelien, noch weiter nach Innen nahmen die Kerne und das Zellprotoplasma an Tinctionsfähigkeit $a b$, es reihte sich eine Langerhan s'sche Körnerschicht an und schliesslich verloren diese gekörnten Zellen ihre Kerne und wurden zu dichtstehenden Lamellen, die sich zu Schüppchen auflösten.

Schmidt erwähnt den Zusammenhang der Epithelperlen mit ihren Plattenepithelien mit den Drüsenschläuchen, die von einschichtigem hohen Cylinderepithel ausgekleidet sind. Er erklärt denselben durch den Durchbruch eines Cystenraumes in einen grösseren Drüsenschlauch.

Nach 10 jähriger Pause beschreibt Kürsteiner2) einen ähnlichen Tumor, welcher bei einer Patientin nach einem Faustschlage gegen die rechte Brust sich langsam entwickelt hatte. Zur Zeit der Operation, 28 Jahre nach dem Trauma, hatte er Kindskopfgrösse erreicht.

Die Schnittfäche der Geschwulst bot einen lappigen Bau, die Lappen liessen meist das Bild des Adenoma phyllodes erkermen; in einzelnen Lappen fanden sich Cysten, welche mit weissbröckliger Masse gefullt waren. In den Partieen mit Cysten und Drüsen waren die Drüsenlumina mikroskopisch von einem einschichtigen Cylinderepithel ausgekleidet; unter demselben war keine deutliche Membrana propria, auch keine zweite

1) Georg Benno Schmidt, Ein l'all von Cystosarkon mit Epithelperlenbildung in der Mamma. Arch. f. Gynäk. Bd. XXIII. 1884. S. 93.

2) W. Kürsteiner, Adenom der Milchdrïse mit cylindrischem und geschichtetem, zum Theil verhorntem lipithel. Virchow's Archiv. Bd. CXXXVI. 1894. S. $30 \mathrm{z}$. 
Ueber Cystofibrosarkume der Mamma mit ejidermoidaler Metaplasie.

Kernlage. An anderen Stellen von ähnlichem Bau constatirte er eine subepitheliale Kernlage. Manchmal fand sich auf dem Cylinderepithel eine Lage von ausserordentlich platten Zellen, mit langen, dunklen, minimalen Kernen. An vielen Stellen, welche das Bild des Adenoma phyllodes ergaben, bestand das Stroma aus sehr lockerem Schleimgewebe.

In den Partieen, in welchen Cysten mit lockerem atheromatösen Inhalt lagen, fanden sich in runden Feldern in weiten Abständen Drüsenbläschen mit geschichtetem Epithel, zum Theil ganz mit demselben angefüllt, zum Theil auch noch mit Lumen versehen, daneben noch solche, die vorzugsweise eine einschichtige Lage von Cylinderepithelien führten; in dieses Cylinderepithel waren aber stellenweise kleine, warzenähnliche Partieen von geschichtetem Plattenepithel eingeschoben. Das Cylinderepithel wurde hier durch das geschichtete emporgehoben. An den "Endbläschen" fand sich zugleich mit dem geschichteten Epithel auch cylindrisches und zwar brach das Cylinderepithel als Wandbekleidung an dem geschichteten entweder scharf ab, oder es ging das cylindrische Epithel auf das geschichtete heriber. Hänfig war das Cylinderepithel von dem unterliegenden geschichteten durch eine schmälere oder breitere Spalte abgehoben, lag sogar manchmal fast ganz frei in dem Lumen.

Die grösseren Cysten mit atheromatösem Inhalt waren zum grössten Theil von verhornten, kernlosen, plättchenartigen Zellen angefuillt, welche sehr locker lagen. Auf der Wand sass eine nur relativ dünne Lage von Epithel; die Zellen derselben waren alle platt gedrückt. Riffzellen oder eine unterste Lage von cylind̈rischen Zellen vermisste $K$ iirs tein er.

In demselben Jahre gab Häckel l) in seinen „Beiträgen zur Kenntniss der Brustdrüsengeschwälste " eine ähnliche Zusammenstellung der einschlägigen Fälle unter Schilderung zweier von ihm untersuchter Tumoren, welche er als "Atherome der Mamma" bezeichnete.

Der erstgesehilderte Tumor trat als selbständige Frkrankung anf, und fasse ich denselben nach den Schilderungen in Uebereinstimmung mit $\mathrm{Häckel}$ als richtiges Atherom auf.

Der zweite Tumor bot einen complicirteren Baı und war vergesellschaftet mit einem Cystosarcoma phyllodes:

Durch den ganzen Bereich der Geschwulst von dem charakteristischen Bau des Cystosarkoms finden sich zerstreut zahllose Cysten von Stecknadelkopf- bis Haselnussgrösse, angefüllt mit ciner weissen Masse, welche in den grösseren sehr regelmässig concentrisch geschichtet ist, so dass die einzelnen Schichten wie Zwiebelschalen um einander angeordnet sind. Dies zusammen mit dem Perlmutterglanz verleiht den Gebilden ein sehr zierliches Aussehen. Sie liegen ohne gesetzmässigge Regelmässigkeit allerwegen in dem Geschwulstgewebe zersprengt.

Mikroskopiseh hat die Wand dieser Cysten il berall eine epidermisartige Beschaffenheit angenommen. Auf einer ditnnen, bindegewebigen Membran, welche allmählig in das umgebende Gewebe ubergeht, sitzt ein vielschichtiges Epithel durchaus von dem Bau der Epidermis, nur dass Papillen, Drtisen und Haare fehlen. Wie die tiefste Schicht des Rete

1) H. H ïckel, Arch. f. klin. Chirurgie. Bd. XLVII. 1894. 
Malpighi, bestebt auch hier die unterste Lage aus cylindrischen Zellen. Dieselben gehen bald in plattere Formen über und bilden Riff- und Stachelzellen, an welche sich allmählig die verschiedenen Processe der Verhornung mit Eleidin- und Keratohyalinkörnern anschliesst.

Als jüngste und letzte Veröftentlichung muss die Arbeit von Hi eb a u m ${ }^{1)}$ genannt werden. Im Prager pathologischen Institut untersuchte er den Tumor einer 44jährigen Frau, welche 11 Partus durchgemacht und 6 Kinder selbst ernährt hatte. Ohne nachweisbare Ursache bemerkte sie die allmählige Entstehung einer Anschwellung in der rechten Mamma, welche sie bald das Krankenhaus aufsuchen liess.

Die amputirte Mamma war $15 \mathrm{~cm}$ lang, $13^{1 / 2} \mathrm{~cm}$ breit, $7 \mathrm{~cm}$ dick. Auf dem Durchschnitt fand sich in dem gelblich-grau verfärbten, ziemlich derben und mit vielen Fortsätzen in den benachbarten Panniculus versehenen Drüsengewebe eine grosse Zahl von hirsekorn- bis erbsengrossen Spalten und Cysten, welche zum grössten Theil mit einer feinkörnigen, dunkelgelben, schmierig anzufühlenden Masse ausgefüllt war. Die Hohlräume selbst erwiesen sich bei der makroskopischen Besichtigung zum Theil als vollkommen glattwandig, zum Theil aber stellten sie Spalten und Höhlen mit vielfachen Ausbuchtungen dar.

Die mikroskopische Untersuchung der Cysten zeigte, dass die Wandungen in ihrem Bau vielfach verschieden waren. In den meisten Fällen erschienen sie ausgekleidet mit einem 1-2 schichtigen abgeplatteten Epithel, während dagegen bei einer ganzen Reihe von Cysten die epitheliale Auskleidung vollständig oder doch zum grössten Theil verloren gegangen war, bei anderen aber wieder aus einem 6-10 schichtigen Platt en e pithel bestand. Die bindegewebigen Cystensepten waren stellenweise stark verdickt, oft aber auch völlig atrophisch geworden. Des Weiteren ergab sich, dass die Cystenbildung aus einer Dilatation sowohl der Milchgänge als auch der Acini und der eigentlichen Ductus galactoferi hervorgegangen war. Von eben beginnender, kaum noch wahrnehmbarer Erweiterung sah man alle Uebergänge bis zur Bildung bohnengrosser Hohlräume. Schliesslich fanden sich an einzelnen entzündlich infiltrirten Partieen und in deren Nähe eine Anzahl von Riesenzellen, welche $\mathrm{H}$ i e b aum als Fremdkörperriesenzellen, veranlasst durch die Usurirung der Cystenwand, ansieht.

Dies sind die wenigen Fälle, welche ich aus der Literatur zusammenstellen konnte.

Wenn ich von den völlig vagen Angaben Porta's absehe, so sind es 14 Autoren, welche sich mit unserer Materie befasst haben. Im Allgemeinen wird von 4 dieser Tumoren Erwähnung gethan,

1) A. Hiebaum, Zur Casuistik der multiplen Cystenbildung in der weiblichen Brustdrüse. Multiple Cysten bei Fibromyom in der Mamilla. Prager med. Wochenschr. 20. Jahrg. 1895. (Diese Arbeit findet - nebenbei bemerkt - keine Erwähnung in 0. Ehrhardt, Ueber Gesehwülste der weiblichen Brustwarze. Deutsche Zeitschrift f. Chirurgie. Bd. I. 1999. S. 373.) 
Ueber Cystofibrosarkome der Mamma mit epidermoidaler Metaplasie.

während wir eine specielle, aber versehieden genaue Beschreibung: an 14 Einzelfällen nunmehr besitzen. Von diesen 14 Einzelfällen waren 4 mit Carcinomen vergesellschaftet - die Angaben ïber diese 4 Tumoren sind alle besonders kurz -; die restirenden 10 Fälle waren fibrosarkomatöser Natur mit diesen epidermoidalen Partieen. ${ }^{1)}$

Schon bei der vorstehenden Aufzählung fällt uns die verschiedene Benennung auf, welche die einzelnen Autoren für die vorliegende Tumorart gewählt haben, ein Zeichen wie verschieden die Auffassung über die Natur derselben ist.

Sehen wir von der Cooper'schen "Hydatidengeschwulst" völlig $a b$, welche Namengebung in den frïheren pathologischen Begriffen seine Erklärung findet, so haben wir eine ganze Reihe (7) von kürzeren Angaben von dem Befund von Epithelperlen besonders in Sarkomen, vereinzelt auch in Carcinomen. Die ausführlicheren Angaben stammen von Model, Schmidt, Kürsteiner, Häckel und Hiebaum.

Nach den mikroskopischen Befunden können wir diese letztgenannten Tumoren nun in zwei Gruppen theilen. Einmal sind es die von Model und Häckel uns bekannt gegebenen, nach deren Schilderung sich in den fraglichen Cysten eine Auskleidung von lediglich Plattenepithelien findet. Daher bezeichnet Model seinen Tumor als "Cystosarkom mit autogenem Atherom" und Hä ckel hält diesen Namen fïr durchaus gerechtfertigt und hat daher in seinen Beiträgen einen besonderen Abschnitt „Das Atherom der Mamma" gebildet, während wir ihn nach unseren Anschauungen für nicht sehr zweckmässig ansehen müssen.

Anders liegen die Verhältnisse in der zweiten zu bildenden Gruppe. Hier finden wir zweierlei Arten von Epithelien vertreten: die normal in der Mamma sich findenden cylindrischen resp. cubischen und daneben Plattenepithelien.

In dem Kürsteiner'schen Tumor finden sich diese beiden Epithelarten in einer theilweise höchst eigenartigen Anordnung, indem das Plattenepithel sich gewissermaassen unter die Cylinderepithelauskleidung sehiebt und letztere dadurch in die Höbe gehoben wird. Daneben finden sich auch Stellen, an denen die Cystenwand lediglich aus epidermoidalen Elementen gebildet ist.

Anders gestaltet sich die Configuration bei den Fällen Hiebaum, B. Schmidt und den meinigen.

1) Nachtrag: Dic „Demonstration eines mannskopfgrossen Sarko-FibroAdenoma mammae mit Cholesteatommassen in den erweiterten Drüsenlumina" wurde von Borrmann-Breslau auf der 71. Naturf.-Vers. in Mïnchen 1899 (Sect. path. anat.) angekündigt, unterblieb aber. 
Ersterer fand eine grosse Anzahl von Cysten lediglich ausgefüllt mit Plattenepithelien: einzelne Cysten dagegen besassen ihre normale cylindrische Auskleidung, während in vielen die verschiedensten Uebergangsformen zwischen beiden Typen vertreten waren.

Dieses Ueberwiegen der epidermoidalen Bestancltheile besteht auch in meinem Il. Tumor. Aber, wie ich dort geschildert habe, finden sich verschiedene Stellen, wo der Uebergang zu Cylinderzellen deutlich zu erkennen ist.

Dieses Verhältniss tritt nun viel deutlicher zu Tage bei dem Fall von B. Schmidt und meinem ersten.

Wie ich schon bei der Schilderung des mikroskopischen Befundes des letzteren erwälnt habe, entsprechen die Bilder, wie sie B. Sch midt in seiner Fig. 4 gegeben, vollständig gewissen Befunden unseres Tumor I. Es sind in ein und derselben Cyste oft beide Epithelarten vertreten. Zuweilen geht die normale Cylinderepithelauskleidung in allmählicher Metaplasie in die l'lattenepithelien über, welche nach geraumem Bestehen in derselben Weise sich wieder in dem Cylinderepithel verlieren. Dann aber haben wir auch schroffe Absätze von Plattenepithel zu Cylinderepithel, welche besonders dort schön zu Tage treten, wo anscheinend ein junger Drüsenschlauch mit typischem Cylinderepithel sich in die Tiefe senkt, wälrend zu beiden Seiten dieser Einsenkung. die Plattenepithellage bis zu ihr herantritt.

Wenn ich nun soeben den Ausdruck Metaplasie gebrauchte, so bin ich mit ihm auf die a etiologische Frage der vorliegenden Tumoren gekommen. Und zwar möchte ich gleich hier die Anschauung vorbringen, welche sich mir bei der Betrachtung im Allswemeinen, dann aber auch beim Studium unserer beiden Tumoren, welche mir in einem glücklichen ergänzenden Verhältniss zu einander zu bestehen scheinen, ergeben liat.

Schon lange ist es bekannt, dass sich auf Schleimbäuten, welche normaler Weise mit Cylinderepithel bedeckt sind, kleine Partieen finden können, welche eine Bedeckung mit Plattenepithelien besitzen.

Derartige Stellen fanden sich vornehmlich im Nasenrachenraum, der Trachea und den Bronchien 1), dann auch in anderen Organen, so in der Auskleidung des Cavum uteri ${ }^{2}$ ) besonders bei chronischen Reizen und auch im Mastdarm"3). Die Kenntniss derartiger Meta-

1) Ernst, Plattenzellenkrebs des Bronchus. Zicgler's Beitrïge. Bd. XX.

2) Flaischlen, Zeitschr. f. Geburtshülfe und Gynäkologie. Bd. XXXII. Gellhorn, Ebend. Bd. XXXVI.

3) Bohm, Virchow's Archiv. Bd. CXL. 
plasieen ist allmählich cine immer grössere geworden, so hat 0 hrl off 1 ) aus dem Greifswalder patholog. Institut einen Beitrag zur Metaplasie des Gallenblasenepithels in geschichtetes Plattenepithel bei Gallenblasencarcinom geliefert.

Zwei neuere cinschlägige Fälle sind von Nehrkorn ${ }^{2}$ ) und Orth ${ }^{3}$ ) mitgetheilt.

Mit einer exquisiten Epithelmetaplasie der Blase, beider Ureteren, sowie eines Theiles der Nierenkelche hat uns Liebenow t) in einer Arbeit aus dem pathologischen Institut zu Marburg bekannt gemacht.

Diese ausgedehnte, mächtige Epithelmetaplasie war in ihrer Entstehung zweifellos anf Steinbildung im Nierenbecken zurückzuführen. Es war zuerst vermuthet worden, dass vielleicht ein Hineinwuchern epidermialer Theile in die Blase und weiter aufwärts stattgehabt habe. Die weiteren Untersuchungen lehrten jedoch, dass eine Metaplasie der vorhandenen Epithelbekleidung sich abgrespielt habe. Interessant ist der Fall noch dadurch, dass sich ein metastatischer Knoten mit Plattenepithelien am Zwerchfell fand, wie ihn Nehrkorn in seiner jüngsten Veröffentlichung auch beschrieben hat.

Wie liegen nun die Verhältnisse bei der Mamma?

Sie ist eine ektodermale Bildung, indem die Drüsenschläuche durch zaptenartiges Hineinwuchern des Ektoderms entstehen. Diese lassen centralwärts ihre soliden Epithelcylinder verhornen und die verhornten Massen werden ausgrestossen, so dass hierdurch die hohlen Drüsenschläuche entstehen.

Auf diesen Modus der Entwicklung gründete G. B. Sch midt seine Annahme, dass in der Zeit der knospenförmigen sccundären Epithelanlage einige, in die Tiefe der Cutis wuchernde Knospen durch irgend welche uns unbekannte Ursachen von dem Mutterboden, aus dem sie entsprossen sind, abgreschnïrt wurden und dort isolirt liegen blieben. Während die Entwicklung der normalen Drïse ilıren Fortgang" nahm, brachten plötzlich durch einen Zufall, welcher die Drüse unter veränderte Ernälırungsbedingungen versetzte, die abgeschnürten versprengten Keime nach Art ihrer Bestimmung verhornte Epithelien hervor, welche eigentlich durch ihren Ausfall eine Lumenbildung

1) Ohrloff, Uober Epitholmetaplasie und Krobsbildung an dor schleinhaut von Gallenblase und Trachea. Dissert. Greifswald 1891.

2) Nehrkorn, Plattenepithelkrebs mit verhomenden Iymphdrüsenmetastasen. Virch. Arch. Bd. CLIV. 1898.

3) Plattenepithelkrebs der Gallenblase. Deutsche med. Wochenschr. Xr. $24.1 \$ 99$.

4) F. Liebenow, Ueber ausgedehnte Epidermisbekleidung der schleimhant der Manwege. Ing.-Ibiss. Marburg 1891. 
herbeiführen sollten, denen aber der Ausgang nach aussen abgeschlossen war. Nun bildeten die abgeschnürten Epidermiszellen so lange polyedrische verhornende Zellen, als es die Räumlichkeit resp. die Elasticität der umliegenden Gewebe gestattete. Die Producte wurden von den Seiten her nach dem Centrum zu geschoben und legten sich zwiebelschalenälınlich aneinander. Da, wo die Umgebung einer solchen Cyste einseitig nachgiebiger war, bauchte sich die so entstehende Epithelperle nach dieser Richtung hin aus. Dehnten sich zwei dicht aneineinander liegende Cystenränme durch starke Production ihrer Wandung weiter und weiter aus und näherten sich gegenseitig immer mebr, so wurde die nach und nach sich verdünnende Zwischenwand allmählich ganz aufgezehrt und es trat so eine Verbindung zwischen beiden und die Bildung einer Biscuitform ein.

Eine zweite Möglichkeit der Entstehung der Geschwulst, welche Schmidt selbst weniger plausibel erscheint, gründet sich auf eine fehlerhafte histologische Construction der Milchgänge. Ihr normaler Weise cylindrisches Epithel soll einige Millimeter unterhalb der Ausmündung in geschichtetes Plattenepithel übergehen. Erstreckte sich nun anormaler Weise das Plattenepithel einige Centimeter statt einige Millimeter in die Tiefe, so könnte die sarkomatös entartete Drüse an der Vorderfläche der Mamma so stark wuchern, dass eine Compression und Abschnürung der Ausfülırungsgänge stattfindet. Dadurch wären dann die verhornten Producte nicht nach aussen entleert, sondern hätten sich vielmehr angesammelt und die Gänge eystisch dilatirt.

Wir müssen Schmidt zureben, dass beide Hypothesen ihre Berechtigung haben. Keine von beiden scheint mir aber völlig zu genügen, um alle Bilder zu erklären, welche er in seinem Falle geschildert und welche, wie erwähnt, auch in unseren beiden Fällen sich wiederfinden.

Es finden sich beide Male nicht nur völlig mit Plattenepithel ausgekleidete Räume, sondern auch Cysten, in denen ein allmählicher Uebergang von Cylinder- zu Plattenepithel besteht neben dem Vorkommen beider Zellarten direct nebeneinander in scharfem Absetzen. An diesen Stellen kann wohl kaum davon gesprochen werden, dass wir abgeschnürte Theile der ursprünglichen Anlage vor uns haben, welche späterhin in Wucherung gerathen sind. Sie bieten auch keinen Anhalt dafür, dass hier etwa ein Durchbruch von einem pathologisch oder atavistisch entarteten Hohlraum in einen normalen benachbarten stattgefunden hat.

Ziehen wir nun aber in Erwägung das, was ich vorher über die 
Metaplasie gesagt habe, so dürfte diese uns unschwer eine Deutung unserer Befunde erlauben.

Hiernach hätten wir es dann mit den progessiven metaplastischen Stadien zu thun und können uns dieselben an den verschiedenen Stellen ausserordentlich gut verdeutlichen.

Einmal haben wir die allerersten Anfïnge der Umwandlung des Cylinderepithels in Plattenepithel.

Wir sehen, wie es stellenweise in seinem metaplastischen Bestreben noch Widerstand findet, an anderen Stellen aber die benachbarten Theile schon mit in den Process hineinzieht. Schliesslich ist derselbe so vollkommen zur Entwicklung gekommen, dass die Cysten völlig in den neuen Zustand versetzt sind und sich nun die prolifere Thätigkeit der neuen Zellgattung entfaltet. Dort, wo die Entfaltung eine mächtige und über weite Bezirke ausgedehnte ist, haben wir dann die Vereinigung mehrerer Räume zu grösseren confluirenden Massen, welche zur Atrophie der Scheidewände und deren Gewebe geführt hat. Schliesslich werden diese Processe so allgemein und so intensiv zur Entwickelung gelangt sein, dass wir von der ursprünglichen Formation wenig oder kaum noch Reste oder Uebergangsformen finden werden, wie in unserm II. Fall, oder dass nur noch Plattenepithelien sich finden wie bei Model, Buch, Hiebaum und im Häckel'schen Fall II.

Der Fall Kürsteiner bot zwar insofern exorbitante Bilder, als die normale Cylinderzellenauskleidung gleichsam abgehoben war durch eine darunter gebildete Schicht von Plattenepithelien. K. enthält sich selbst jeder Deutung dieser Bilder. In welcher Epithellage, besonders bei den mehrschichtigen eubischen Auskleidungen der metaplastische Process zuerst einsetzt, ob lediglich, wie man zunächst vielleicht annehmen könnte, in den oberen oder nicht auch in tieferen, darüber sind wir nicht näher unterrichtet und haben auch meine Präparate keinen Anhaltspunkt gegeben. Wir wissen aber, dass bei anderen Processen, so bei der Hautregeneration die unterste Lage der Epiderniszellen, die sogenannte Keimschicht, eine Hauptrolle spielt.1)

Kürsteiner selbst glaubt den Gedanken der Herausbildung des geschichteten Epithels aus dem Cylinderepithel durch das erwähnte Verhalten beider Epithelformen abweisen zu müssen; er schildert aber des Genaueren eine subepitheliale Kernlage und erwägt, ob das geschichtete Epithel nicht aus dieser hervorgegangen sei.

1) Fnderlen, Histologische Untersuchungen über die Einheilung von Pfropfungen nach Thiersch und Krause. Deutsche Zeitschrift für Chirurgie. XLV. Bd. 
Sollte dies der Fall sein, so würden wir es, wenn auch mit einer modificirten Form der Metaplasie zu thun haben.

Wenn in den Tumoren von Model, Buch, Hiebaum und Häckel sich schliesslich gar keine Andeutungen der ursprünglichen cylindrischen Auskleidung finden, so könnten wir annehmen, dass die Metaplasie eben in allen Theilen schon völlig sich vollzogen hat, und wir vor dem Endfactum stehen.

Nach diesem Allen halte ich den zuerst von Nodel (1858) eingeführten und später von Häckel acceptirten Namen „Atherom der Mamma" für diese Art der Tumoren nicht für zutreffend.

Der Name Atherom ist zur Zeit im Gebrauch für diejenigen Gebilde, welche wir bisher als entstanden ansahen durch eine richtige Retention in Talgdrüsen oder Haarbälgen, die zu cystischer Erweiterung führten. Nach Chiari's 1) Untersuchungen wissen wir jetzt, dass es sich bei den Atheromen immer um eine congenitale Anlage bandelt, ähnlich wie bei den Dermoiden.

Formen des metaplastischen Uebergangs wie bei mseren Tumoren sind bei ihnen überhaupt nicht vorhanden, da eben immer die $\mathrm{Mn}$ lehnung an den Charakter der äusseren Haut dauernd bestanden hat.

Auch die Mächtigkeit der Entwickelung, die Multilocularität und Multiplicität der veränderten Cysten in der Mamma stimmen nicht mit dem Bilde der eigentlichen Atherome ïberein.

Die Möglichkeit des Vorkommens von echten Atheromen an der Mamma muss ja zugegeben werden. Ich habe aber unter den in Frage kommenden Fällen keinen einwandsfreien finden können. Man könnte zunächst vielleicht geneigt sein, den von Häckel unter den Atheromen als ersten Fall angeführten Befund hicrher zu rechnen; bei genauerer kritischer Würdigung erscheint die Deutung aber zweifelhaft.

Es handelt sich um eine 25 jährige Frau, welche vor 1//2 Jahren geboren hatte und im Wochenbett zufällig einen harten, wallnussgrossen, scharfbegrenzten Knoten in der linken Manma bemerlite. Makroskopisch erwies er sich als eine mit graugelben, knetbaren Massen gefüllte Cyste, welche mikroskopisch eine dünne bindegewebige Membran besass, auf der eine 3-4 schichtige Lage von cubischen Epithelien sich fand, welche allmählich undeutliche Kernfärbung annahm und schliesslich in unregelmässige Schollen überging:

Nach der makro- wie mikroskopischen Beschreibung glaube ich aber, dass dieser Tumor weniger für ein richtiges Atherom anznsehen

1) H. ('hiari, Ueber dic Genese der sogen. Atheromeysten. Zeitschrift f. Heilk. XII. 1891. 
ist, sondern dass wir es hier eher mit einer Retention in einem Ausführungsgang zu thun haben.

Diesen Atheromen nahestehend sind Bildungen der Gruppe "Dermoide", von denen Herrman $n^{1}$ ) eine Schilderung und Zusammenstellung gegeben hat. Der von ihm beschriebene Tumor zeichnete sich unter anderem dadurch von den unsrigen aus, dass sich eine gute Ausbildung von richtigen Papillarkörpern fand, welche wir in allen von mir aufgezählten Tumoren vermissen, so dass die Bemerkung von B. Schmidt, dass wir „ein vollständiges Gepräge der äusseren

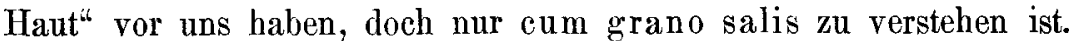

Nach den Befunden und meinen vorstehenden Auslassungen glaube ich daher, dass man nicht die vorliegenden Tumoren mit einem bestimmten Namen wie Atherom, Cholesteatom oder ähnlichen bezeichnen soll, welche Benennungen zur Zeit ganz bestimmt umschriebenen Geschwulstgruppen zukommen. Ich wählte die Bezeichnung, welche primär der Tumorentwicklung entsprach - in unseren beiden Fällen und den anderen genau beschriebenen dem Cystofibrosarkom mit dem ergänzenden Zusatze der "epidermoidalen Metaplasie".

Suchen wir schliesslich noch einen Anhalt für die Veranlassung der Metaplasie zu gewinnen, so sehen wir einmal, dass dieselbe immer vergesellschaftet ist mit einer anderweitigen Geschwulstbildung der Mamma und zwar, wie eben erwähnt, meist mit dem Cystosarkom mit seinen mannigfaltigen varïrenden Bildern.

Wahrscheinlich ist, dass diese Geschwulstwucherung den Anstoss zur Einsetzung der Metaplasie gegeben, obgleich es immerhin auffallend ist, dass bei der relativen Häufigkeit derselben wir metaplastische Processe so eminent selten finden.

Klinisch wird meist angegeben, dass ein Trauma früher statt gefunden hat:

Cooper giebt einen Stoss in seiner Anamnese an. Bei Model erfolgte das Trauma in Gestalt des Stosses einer Kuh auf den allerdings schon 3 Jahre bestehenden Tumor, woranf aber ein besonders rasches Wachsthum erfolgte. Bei Schmidt war eine ausserordentlich stürmische Umarmung mit Druck der betreffenden Mamma anamnestisch erwähnt, während bei Kürsteiner ein allerdings 22 Jahre zurückliegender Faustschlag aetiologisch beschuldigt wird.

Derartige Angaben sind zwar immer sehr vorsichtig aufzufassen, müssen aber bei unserer totalen Unkenntniss berücksichtigt werden,

1) K. Herrmann, Ueber einen Fall von Dermoidcyste in der rechten Mamma. Prager med. Wochenschrift. 15. Jahrg. 1890. \$. 547. 
umsomehr da bei den Eingangs erwähnten metaplastischen Processen in der Gallenblase, im Uterus oder Respirationstractus verschiedene Reize, sei es mechanischer Art (durch Gallen- resp. Nierensteine), sei es chemisch-medicamentöser Art nachweislich eine wichtige Rolle gespielt haben.

Werfen wir zuletzt noch einen Blick auf das klinische Material, so sehen wir die Tumoren bei Personen verschiedensten Alters auftreten: vom 27. bis 72. Lebensjahr sind Patienten vertreten. Und nicht nur dies. Selbst beim männlichen Geschlecht finden wir diese seltenen, unserm Verständniss jetzt vielleicht etwas näher gerückten, aber in Manchem doch noch unklaren Geschwülste.

\section{Erklärung der Abbildungen anf Tafel III.}

Figur I. Senkrechter Durchschnitt durch den Tumor in der Höhe der Mamilla (vgl. S. 69).

$m=$ Mamilla.

$b=$ bindegewebige Kapsel.

$c=$ cystische Hohlräume.

$p=$ papilläre Excrescenzen in die Cysten.

$e=$ Epithelperlen.

$h=$ Hämorrhagie.

Figur II. Schnitt parallel zu dem ersten ea. $3 \mathrm{~cm}$ entfernt.

Dieselben Bezeichnungen wie Fig. I.

$f=$ Stellen, wo mehrere Epithclperlen confluirt sind.

$e_{1}=$ Epithelperlen an einer hämorrhagischen Stelle gelegen. 